\title{
Automatic Mosquito Control Device Using Infrared Motion Sensor
}

\author{
Akiti Kenneth Tetteh Li Shuguang \\ School of Automation, Zheijang University of Science and Technology, Hangzhou, China
}

\begin{abstract}
Malaria is a disease transmitted by female Anopheles mosquitoes to humans. Mosquitoes are a carrier of malaria. There are many types of vector control strategies, including physical, chemical, and biological methods. Individuals, local communities, and more organized public health vector management programs often need to work together to effectively identify and control mosquito farms. Efforts should be made to destroy mosquito eggs and reduce the density of larvae and adults. The World Health Organization recommends integrated vector management (IVM) as a strategy to improve vector control. IVM combines good practices that maximize an effective, safe, environmentally friendly, and cost-effective approach to disease vector control. Effective and well-tested vector control strategies include: Biological control: larvae and biolarvicidal fish, such as mosquitoes in ditches and ponds or other predators to control mosquito larvae. Chemical control: larvae and adults (indoor spray residues, insecticide-treated bed nets, etc.). Adult mosquitoes are small vulnerable insects with slender bodies, a pair of slender wings and three pairs of thin long legs. Mosquito control can be improved by using an automatic mosquito control device with an infrared motion sensor. Suppression of malaria vectors is an important means of preventing and reducing malaria transmission. To reduce malaria, mosquitoes in and around our environment must be controlled. The introduction of an infrared motion sensor in an automatic mosquito control device would eliminate the reduction in the number of mosquitoes in our environment. Add an infrared motion sensor to the automatic mosquito control device for easier mosquito control in our homes and environments. To customize automatic mosquito control by adding an infrared motion sensor.
\end{abstract}

DOI: $10.7176 /$ ISDE/12-3-01

Publication date:September $30^{\text {th }} 2021$

\subsection{INTRODUCTION}

Malaria is a disease that is transmitted among human by female Anopheles mosquito. Mosquitoes become the vector for malaria. Many types of vector control strategies exist including physical, chemical, biological methods. Individuals, local communities, and larger organized public health vector management programmes frequently need to work together to effectively identify and control mosquito breeding sites Common breeding habitats include common household containers (used for domestic water storage and decorative plants) as well as rain-filled habitats (used tyres, discarded food and beverage containers, blocked gutters and construction sites). Efforts must be made to destroy mosquito eggs and reduce larval and adult densities ${ }^{(1)}$.The World Health Organization recommends Integrated Vector Management (IVM) as a strategy to improve vector control. IVM is a decision-making process for the management of vector populations, so as to reduce or interrupt transmission of vector-borne diseases. IVM combines common-sense practices that maximize effective, safe, environmentally sensitive and economical approaches to control disease vectors. IVM is based on ecological, economic and social criteria and integrates multidisciplinary methodologies into pest management. Effective, well tested vector control strategies include:

(i)Environmental Management: source reduction and habitat modification / manipulation, for example digging ditches and covering ponds in the targeted marshlands;

(ii) Mechanical Control: screening window and doors, drilling holes in fenders and removal and safe storage of scraps;

(iii) Biological Control: larvivorous fish and bio-larvicides, for example mosquito fish in ditches and ponds or other predators to control mosquito larvae.

(iv)Chemical Control: Larvicides and Adulticides (Indoor Residual Spraying, Insecticide Treated Bed-nets, etc. $)^{(1)}$.

One of the most important advances in the control of mosquito was the development of insecticides that remain active over extended periods. Spraying with residual insecticide became particularly important in the control of the mosquito vectors of malaria, which often rest on walls before and after feeding ${ }^{(2)}$.

Adult mosquitoes are small, fragile insects with slender bodies, one pair of narrow wings and three pairs of long, slender legs. They vary in length from $3 / 16$ to $1 / 2$ inch $(5 \mathrm{~mm}$ to $13 \mathrm{~mm})$. Mosquitoes have an elongate proboscis with piercing mouthparts with which the female bites and feeds on blood ${ }^{(3)}$. The control of mosquito can be enhanced by the use of an automatic mosquito control device with infrared motion sensor. This device will automatically spray as the mosquito is been attracted and its motion is sensed. This device can be used when the room light is on or off to the comfort ability of the user. Malaria is a life threatening disease caused by 
plasmodium parasites that are transmitted to people through bites from infected female Anopheles mosquitoes. These mosquitoes are also known as Malaria vectors. Malaria vector control is the main way to prevent and reduce Malaria transmission. Due to poor management of waste in our environment, there is an enhancement to the increase in reproduction of these Malaria vectors in our homes.

\subsection{Motivation}

The need for control of mosquitoes in our environment and surroundings is necessary for the reduction of malaria. By the introduction of infrared motion sensor into an automatic mosquito control device, the reduction of mosquito would be achieved in our environment. To add an infrared motion sensor to an automatic mosquito control device for easier termination of mosquitoes in our homes and environment. To modify an automatic mosquito control by adding an infrared motion sensor. To obtain easy and much better detection and termination of mosquitoes.

\subsection{LITERATURE REVIEW}

The following are the previous work done by the researchers on mosquito control measures and devices. Paul Garnel, et al. (2009) presented a research document on malaria which had caused several death cases, mostly in children that are below the age of five. It stated a means of malaria control through mosquito control. This means of mosquito control was by the use of mosquito net which was treated with insecticide. And the result was successful in preventing malaria by sleeping under the treated mosquito net. From the use of the insecticide treated mosquito net to prevent malaria in children, statistics was presented which showed the reduction of death rate in children. Jerome Goddard (2003) presented a paper on Setting Up A Mosquito Control Program. This paper talks about the various ways in which mosquito control were subjected to different changes. He went ahead to explain how the construction of man- made lakes and the use of irrigation agriculture have increased the numbers of many mosquitoes such as malaria mosquitoes (Anopheles) and the dark rice field mosquito (Psorophoracolumbiae). Mosquito control strategies have changed considerably over the past few decades. Diesel oil and inorganic poisons such as Paris green (copper-aceto-arsenite) were the basic tools of early mosquito larva control efforts. And it also tells us that, presently, only a few of these biological control agents, including the bacteria Bti (Bacillus thuringiensisisraeliensis) and mosquito fish (Gambusiaaffinis), are commercially available. Barbara P. Ogg, et al. (2010) presented a paper on Residential mosquito control. This paper presented to us that the most important element in mosquito control is the elimination of standing water, which serves as a breeding site for mosquitoes in our environments. Also made mention of the use of personal repellents, bug zappers, ultrasonic devices, mosquito repellent plants for mosquitoes. All these measures all sums up to the control of mosquito in our environment leading to the reduction of malaria outbreak in our society. Sindhu. A.M, et al. (2016) presented a paper on Smart Street light using Infrared motion Sensor. This paper presented to us that the main aim of Smart Street light is to reduce the power consumption when there is no vehicle movement on the road. The smart street light will turn to be on when vehicles are on the road and off if there is none. And most importantly, it presented that using Infrared motion sensor, the smart street light provided a solution for energy saving which is achieved by sensing an approaching vehicle. With the use of infrared motion sensor, it is possible to switch on block of street light ahead of a vehicle. Jaeseok and Sang-Shin Lee (2014) presented a paper on Human Movement Detection and Identification Using Pyroelectric Infrared Sensors. This paper made known to us that pyroelectric infrared sensors have been widely used for human tracking system. And that a dense array of pyroelectric infrared sensors has digital output and the modulated visibility of Fresnel lenses that can provide capabilities for tracking human motion. This paper also presented to us a situation where a pyroelectric infrared sensor having two sensing elements aligned in motion plane and its output signal captured in the case of walking in different direction, at different distances, and at different speed levels. As observed in the literature review above, the previous work done on the control of mosquitoes has been proven to be effective in their various areas. With our device, this is adding an infrared motion sensor to an automatic mosquito spray, would further help in the detection and termination of mosquitoes in our environment and reduction of malaria transmission.

\subsection{MATERIALS AND METHODOLOGY}

3.1 Materials

The following are the components, specifications and quantity that were used in the assembling of an automatic mosquito control device using infrared motion sensor. 
Table: 3.1 Development Materials

\begin{tabular}{|l|l|l|}
\hline Component & Specification & Quantity \\
\hline Microcontroller & ATMEGA328P & 1 \\
\hline Diode & 1KAB100E & 4 \\
\hline Transformer & SM-R6 & 1 \\
\hline Voltage regulator & LM7805 & 1 \\
\hline LCD display & JHD_162ALCD (16x2 LCD) & 1 \\
\hline Capacitors & $2200 \mu \mathrm{F}, 100 \mu \mathrm{F}, 22 \mathrm{pF}$ and 22PF & 4 \\
\hline Resistors & $1 \mathrm{~K}, 10 \mathrm{k}$ & 2 \\
\hline Infrared motion sensor & pyroelectric sensor & 1 \\
\hline Automatic spray & Deogroup dispenser & 1 \\
\hline Transistor & $2 \mathrm{~N} 2222$ & 1 \\
\hline Variable resistor & $10 \mathrm{k}$ & 1 \\
\hline LED & Red & 1 \\
\hline Crystal oscillator & $16 \mathrm{MHz}$ & 1 \\
\hline
\end{tabular}

3.2 Methods and Processes

The systematic precise and comprehensive procedure employed in the design and construction of the device is given below. It includes the block diagram of the design with circuit diagram and calculation. It addresses the various objective of the project and explanation of all functional block.

3.2.1 Block Diagram of the System

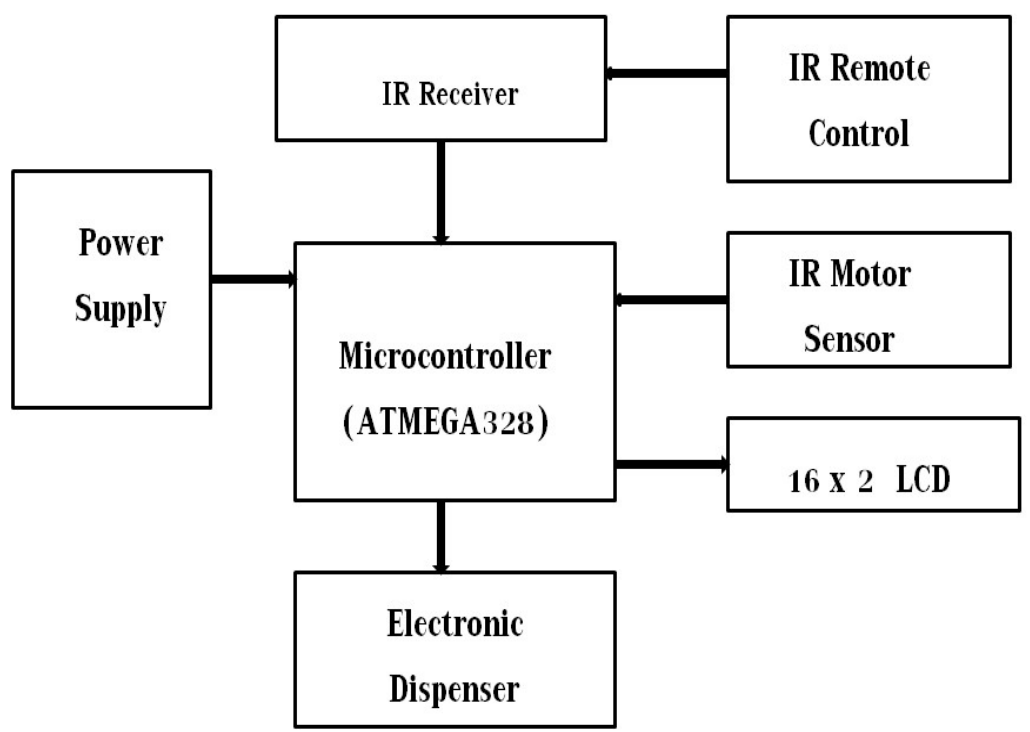

3.2.1 Description of Block Diagram

Figure 1: Block Diagram of the System.

Figure 3.1 is the block diagram of the automatic mosquito control device using infrared motion sensor comprises of the power supply unit, where a voltage of $5 \mathrm{v}$ is produced for that units. This power supply involves a transformer, a bridge rectifier and a voltage regulator. The power supply unit gives a constant $5 \mathrm{v}$ to the microcontroller. The unit of the microcontroller involves atmega328p, a transistor, a crystal oscillator and two 22PF capacitor connected to the crystal oscillator. The microcontroller unit controls other unit such as the LCD unit, electronic dispenser unit, infrared motion sensor unit, infrared remote receiver unit. When the infrared motion sensor senses the motion of a mosquito, it sends signal to the microcontroller unit which immediately sends signals to LCD and electronic dispenser. Then the LCD displays and the dispenser sprays. The infrared remote receiver receives signal from remote, the receiver then send signal to the microcontroller for increment or decrement of time interval for the dispenser to spray.

\subsection{Design}

The automatic mosquito control device is designed to very small amount of power. The voltage requirement of every major component in the circuit is within the range of $1.8 \mathrm{v}-12 \mathrm{v}$. Therefore for convenience, a $5 \mathrm{v}$ DC power supply is used to drive the circuit. 


\subsection{Circuit Diagram of the System}

The figure 3.2 reviews the circuit connection of the system. How each material is been placed and connected on the printed circuit board?

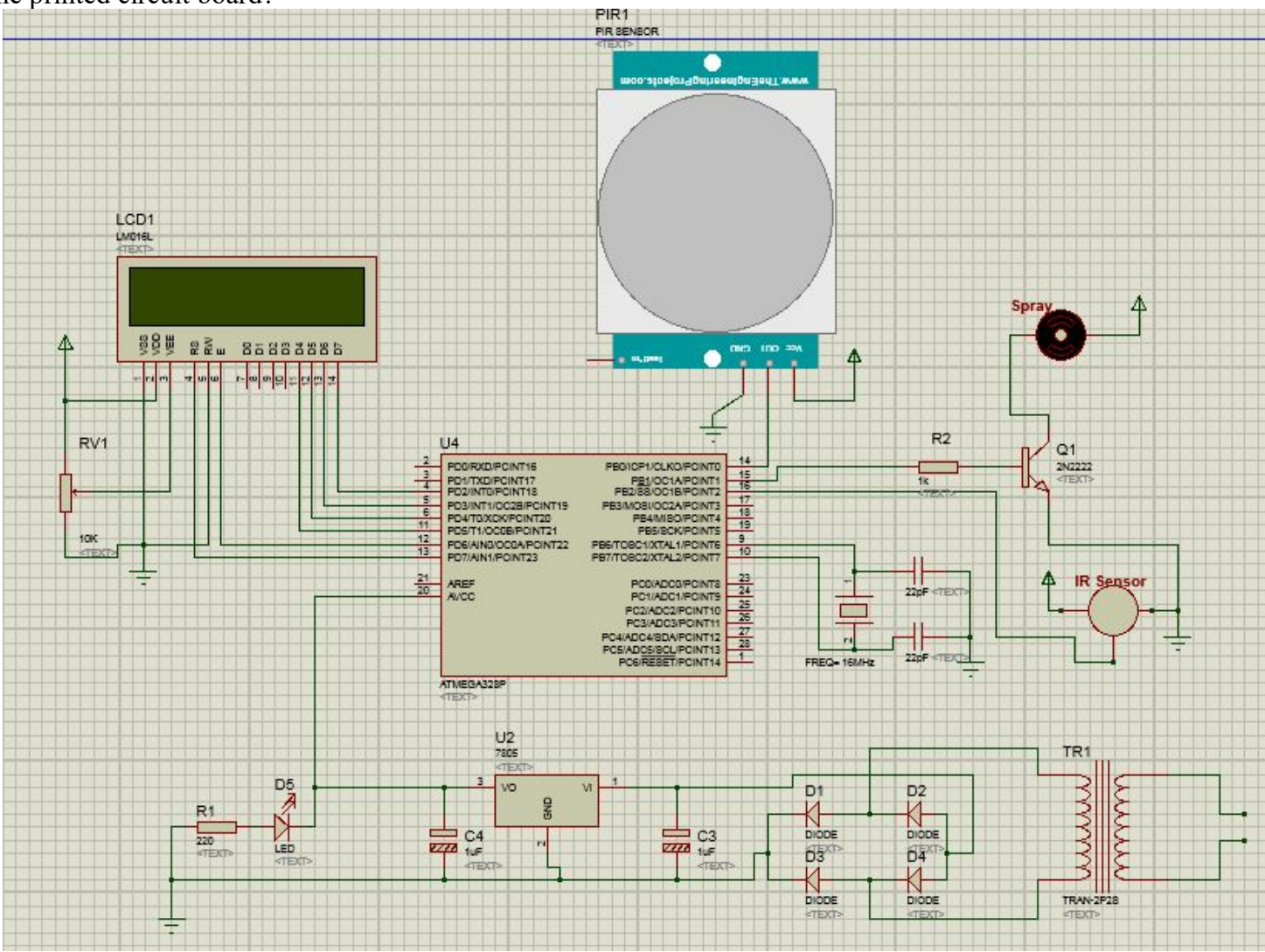

Figure 2: Schematic Circuit Diagram of Automatic Mosquito Control System

\subsubsection{Power Supply}

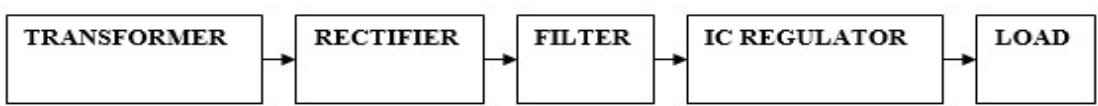

Figure 3: Power Supply Process

The AC voltage, typically $220 \mathrm{~V}$ RMS, is connected to a transformer, which steps down that AC voltage to $12 \mathrm{v}$ AC output. A bridge rectifier then provides a full-wave rectified voltage that is initially filtered by a simple capacitor filter to produce a dc voltage. This resulting dc voltage usually has some ripple or ac voltage variation. A filter circuit removes the ripples and the regulator circuit remains the same as dc value at the output even if the input dc voltage varies. This voltage regulation is usually obtained using one of the popular voltage regulator IC units. This project required $5 \mathrm{v}$; hence the voltage regulator LM7805 IC unit is used produces the constant 5v needed by the microcontroller. 


\subsubsection{Microcontroller}

\begin{tabular}{|c|c|c|c|}
\hline \multirow{2}{*}{ 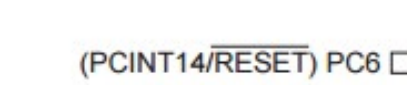 } & \multicolumn{2}{|c|}{$\circlearrowright$} & \multirow[b]{2}{*}{ 口 PC5 (ADC5/SCL/PCINT13) } \\
\hline & 1 & 28 & \\
\hline (PCINT16/RXD) PD0 Д & 2 & 27 & D PC4 (ADC4/SDA/PCINT12) \\
\hline (PCINT17/TXD) PD1 ㅁ & 3 & 26 & D PC3 (ADC3/PCINT11) \\
\hline (PCINT18/INT0) PD2 - & 4 & 25 & P PC2 (ADC2/PCINT10) \\
\hline (PCINT19/OC2B/INT1) PD3 Б & 5 & 24 & P PC1 (ADC1/PCINT9) \\
\hline (PCINT20/XCK/TO) PD4 & 6 & 23 & 口 PC0 (ADC0/PCINT8) \\
\hline VCC & 7 & 22 & PGND \\
\hline GND $\square$ & 8 & 21 & $\square$ AREF \\
\hline (PCINT6/XTAL1/TOSC1) PB6 Д & 9 & 20 & $\sqsupseteq$ AVCC \\
\hline (PCINT7/XTAL2/TOSC2) PB7 문 & 10 & 19 & 口 PB5 (SCK/PCINT5) \\
\hline (PCINT21/OC0B/T1) PD5 Д & 11 & 18 & D PB4 (MISO/PCINT4) \\
\hline (PCINT22/OCOAVAIN0) PD6 Б & 12 & 17 & D PB3 (MOSI/OC2A/PCINT3) \\
\hline (PCINT23/AIN1) PD7 口 & 13 & 16 & 口 PB2 (SS/OC1B/PCINT2) \\
\hline (PCINTO/CLKO/ICP1) PBO Д & 14 & 15 & 口 PB1 (OC1AVPCINT1) \\
\hline
\end{tabular}

Figure 4: Pin Diagram of the Microcontroller ATMEGA328.

Microcontroller is a general purpose device, which integrates a number of the components of a microprocessor system on to single chip. It has inbuilt CPU, memory and peripherals to make it as a mini computer. A microcontroller also called an embedded controller because the microcontroller and its support circuits are often built into, or embedded in, the devices they control. The automatic mosquito control device uses an infrared motion sensor is using ATMEGA328 microcontroller. The pin diagram is show in fig 3.3 above and it has the following features:

$>$ 28-pin AVR Microcontroller

$>$ Flash Program Memory: 32 kbytes

$>$ EEPROM Data Memory: 1 kbytes

> SRAM Data Memory: 2 kbytes

> I/O Pins: 23

$>$ Timers: Two 8-bit / One 16-bit

$>$ A/D Converter: 10-bit Six Channel

> PWM: Six Channels

$>$ RTC: Yes with Separate Oscillator

$>$ MSSP: SPI and $I^{2} C$ Master and Slave Support

$>$ USART: Yes

$>$ External Oscillator: up to $20 \mathrm{MHz}$

The microcontroller is the heart of this project. It is programmed in a way that it is able to give out signals to various unit at appropriate times.

\subsubsection{Infrared Motion Sensor}

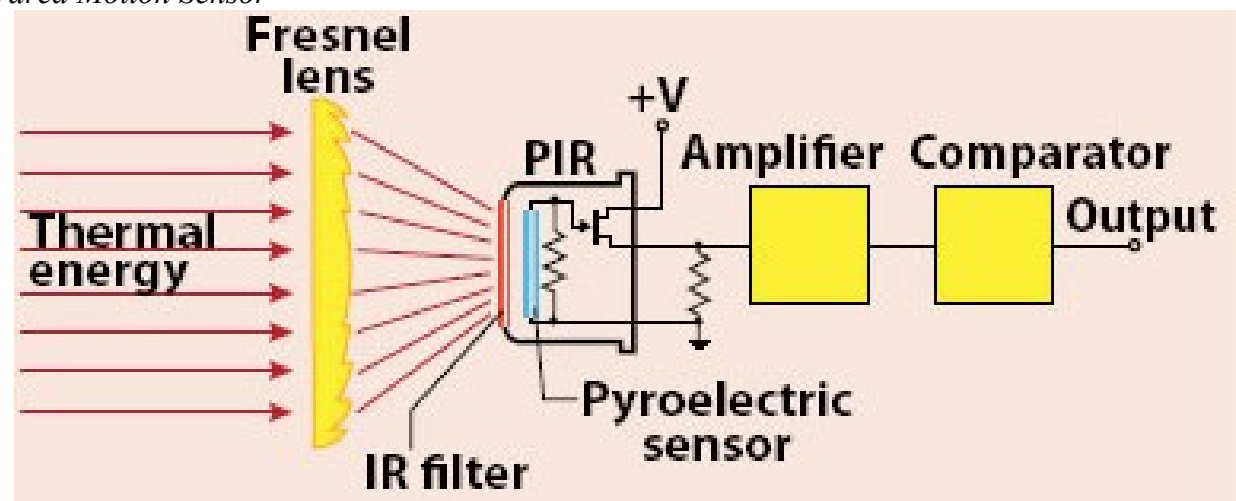

Figure 5: Passive Infrared Motion Sensor Block Diagram.

The infrared motion sensor senses the motion of the mosquitoes in this project. They are often referred to as passive infrared, pyroelectric sensor or infrared motion sensor. Infrared motion sensors are basically made of pyroelectric sensors which can detect levels of infrared radiation. Everything emits some low level radiation and the hotter something is, the more radiation emitted the sensor in the motion detector is actually split in two halves. The reason for that is that we are looking forward to detect motion (change) not average infrared 
radiation levels. The two halves are wired up so that they cancel each other out. If one half sees more or less infrared than the other, the output will swing high or low.

3.4.5 Spray Dispenser

This is an apparatus for periodically activating a control valve on a canister to discharge a quantity of the contents of the canister into a room, the apparatus comprise of the following: an actuator which engages the canister to open the control valve, an electric motor which has an output shaft that engages a plurality of gears that engages the actuator to work. The combination of the motor, the gears and the actuator engages the canister to open the valve. The motor is electrically connected to the transistor which is also connected to the microcontroller for signals. The microcontroller periodically activates the actuator at intervals that it receives signals from the infrared motion sensor corresponding to the motion sensed interval. When a determination is made that the motion of the mosquito is detected, and when a determination is made that the no motion sensed such as no mosquito detected, the controller of the dispenser does not activate the actuator and it does not dispense.

3.4.6 Infrared Remote Recevier

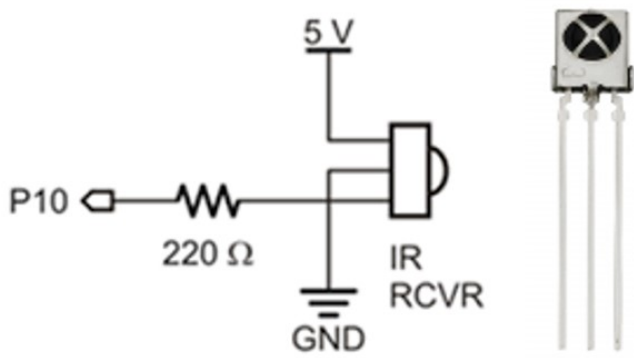

Figure 6: IR Sensor and its Circuits connection

An IR receiver can detect bursts of infrared light sent by a common remote controller (like for a television), and then output a pattern of high/low signals to a Propeller I/O pin. The infrared receiver needs three connections: $5 \mathrm{~V}, \mathrm{GND}$, and an $\mathrm{I} / \mathrm{O}$ pin to read the signal it sends. The circuitry inside the infrared receiver makes it safe to connect its signal pin to the Propeller I/O pin with a small resistor, even though the sensor is powered by $5 \mathrm{~V}$.

3.4.7 CALCULATION

According to the data sheet of the manufacturer, $\beta=75$

$\mathrm{R}=1000=1 \mathrm{k} \Omega$

$\mathrm{V}_{\mathrm{BB}}=5 \mathrm{~V}$

$\mathrm{I}_{\mathrm{B}}=\mathrm{V}_{\mathrm{BB}} / \mathrm{R}$

$\mathrm{I}_{\mathrm{B}}=5 \mathrm{~V} / 1000=0.005$

$\mathrm{I}_{\mathrm{B}}=5 \mathrm{~mA}$.

The relationship between the collector and the base is given by

$\mathrm{Ic}=\beta \mathrm{I}_{\mathrm{B}}$

$\mathrm{I}_{\mathrm{C}}=75 \times 0.005$

$\mathrm{I}_{\mathrm{C}}=0.375$

$\mathrm{I}_{\mathrm{C}}=375 \mathrm{~mA}$ 


\subsection{Flow Chart of the System}

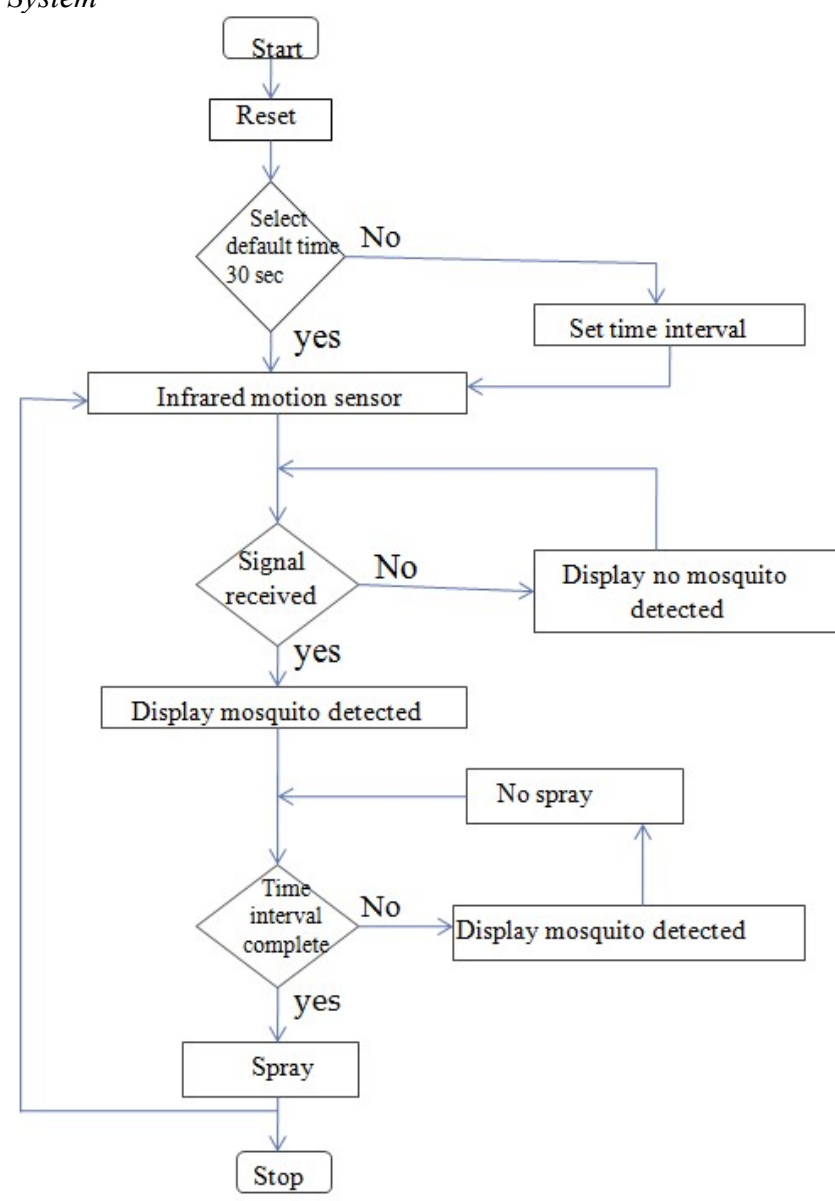

Figure 7: Flow Chart of the System

When the system is switched on, it will reset itself. Then operate in default time interval which 30 seconds. If the time is appropriate for the user, he or she can allow it to continue. But if not appropriate, the user is allowed to increase or decrease the time using the remote. The operation depends on the infrared motion sensor. When signal of motion is received, the LCD will display "mosquito detected" and if the signal is not received, it will display "no mosquito detected". When the time interval is complete, the dispenser sprays else it displays mosquito detected but will not spray until the time interval is complete and signal is received. And after that spray, it has to depend on the infrared motion sensor to receive signal and perform it function.

\subsection{RESULT AND ANALYSIS}

The table below shows the result of the design, where it was tested at various location and at different period of time.

Table 4.1 System Results

\begin{tabular}{|l|c|c|c|c|}
\hline \multicolumn{1}{|c|}{ Room Outlet } & Period of 30 Minutes & Period of 1 Hours & Period of 2 Hours & Total \\
\hline Hall & 5 & 10 & 7 & 22 \\
\hline Bedroom & 7 & 13 & 8 & 28 \\
\hline Bathroom & 10 & 15 & 4 & 29 \\
\hline
\end{tabular}

\subsection{Analysis}

It was observed that when the device was placed in the hall for the period of 30 minutes, five mosquitoes were found dead. Again when allowed for an hour, 10 were found dead. Then it was allowed for two hours and 7 were found dead. The same goes for the bedroom and the bathroom respectively according to table 4.1. This shows that when the device in been introduced for the first few minutes it terminated few. When the time is been increased to an hour it terminated more. And that when it the time was increased more, the number terminated reduced because the device has actually been effective in killing the mosquitoes. 


\subsection{Conclusion and Recommendation}

The automatic mosquito control device using an infrared motion sensor was successfully designed with the used of few electronic materials built in accordance to the circuit diagram which has resulted in a successful output as the infrared motion sensor was able to detect mosquitoes and the microcontroller used that signal to enable the dispenser to spray. This project is recommended to every one for the control of mosquitoes in their homes so as to reduce the change of being bitten by mosquito which results in malaria. The project seeks to terminate mosquitoes in our rooms. This is designed to be placed in a room and on the wall. It should be switch on only when there is no individual in the room due to the fact that the chemicals in the canister might affect the individual's health. Malaria is a complex disease. Its severity is a function of the interaction between the parasite, the Anopheles mosquito vector, the human host and the environment. The risk of malaria infection is determined by the number of vectors, their survival rate.

\section{Reference}

1. UN MEDICAL SERVICES DIVISION 2016Best Management Practice for Mosquito Control Program for UN Duty Stations Updated on 23 February

2. Fontaine, R.E, World Health Organization. House-spraying with residual insecticides, 1978

3. Mosquito Pest Management William F. Lyon Extension Entomologist Julie A. Steele Research Assistant Ohio State University Extension Entomology Building 1991 kenny Road Columbus, Ohio 43210- 1090.

4. Systematic Reviews in Malaria: Global Policies Need Global Reviews Paul Garner, MB, BS, MD, FFPHMa, ${ }^{*}$, Hellen Gelband, MHSb, Patricia Graves, MSPH, PhDc, Katharine Jones, MBChB, MRCGP, DTM\&H a, Harriet MacLehose, BSc, PhD, MAa, PieroOlliaro, MD, PhD d, on behalf of the Editorial Board, Cochrane Infectious Diseases Group.

5. Setting Up A Mosquito Control Program By Jerome Goddard, Ph.D. Medical Entomologist Bureau Of General Environmental Services Mississippi State Department Of Health, P. O. Box 1700 Jackson, Mississippi 39215-1700 601-576-7689 Updated June 2003

6. Residual Mosquito Control by Babara P. Ogg, Extention Educator; Frederick P Baxendale, Extention Entomolonomist; and James A Kalish; Extention Associate. Index Insect and Othet Pest. Issued September 2010

7. Sindhu. A.M, Jerin George, Sumit Roy, Chandra J: Smart Streetlight Using IR Sensors. Doi: 10.9790/005003023944. e-ISSN: 2394-0050, P-ISSN: 2394-0042.Volume 3, Issue 2. (Mar. - Apr. 2016), PP 39-44

8. Jaeseok Yun and Sang-Shin Lee. Human Movement Detection and Identification Using Pyro electric Infrared Sensors. 2014 May 5. doi: 10.3390/s140508057 PMCID: PMC4063065 